\title{
Gravitational Collapse in Husain space-time for Brans-Dicke Gravity Theory with Power-law Potential
}

\author{
Prabir Rudra1 \\ Department of Mathematics, Pailan College of Management and Technology, Bengal Pailan Park, Kolkata-700 \\ 104, India.
}

\begin{abstract}
Ritabrata Biswas 2
Department of Mathematics, Indian Institute of Engineering Science and Technology, Shibpur, Howrah-711 103, India.
\end{abstract}

\author{
Ujjal Debnath ${ }^{3}$ \\ Department of Mathematics, Indian Institute of Engineering Science and Technology, Shibpur, Howrah-711 \\ 103, India.
}

\begin{abstract}
The motive of this work is to study gravitational collapse in Husain space-time in Brans-Dicke gravity theory. Among many scalar-tensor theories of gravity, Brans-Dicke is the simplest and the impact of it can be regulated by two parameters associated with it, namely, the Brans-Dicke parameter, $\omega$, and the potential-scalar field dependency parameter $n$ respectively. V. Husain's work on exact solution for null fluid collapse in 1996 has influenced many authors to follow his way to find the end-state of the homogeneous/inhomogeneous dust cloud. Vaidya's metric is used all over to follow the nature of future outgoing radial null geodesics. Detecting whether the central singularity is naked or wrapped by an event horizon, by the existence of future directed radial null geodesic emitted in past from the singularity is the basic objective. To point out the existence of positive trajectory tangent solution, both particular parametric cases(through tabular forms) and wide range contouring process have been applied. Precisely, perfect fluid's EoS satisfies a wide range of phenomena : from dust to exotic fluid like dark energy. We have used the EoS parameter $k$ to determine the end state of collapse in different cosmological era. Our main target is to check low $\omega$ (more deviations from Einstein gravity-more Brans Dicke effect) and negative $k$ zones. This particularly throws light on the nature of the end-state of collapse in accelerated expansion in Brans Dicke gravity. It is seen that for positive values of EoS parameter $k$, the collapse results in a black hole, whereas for negative values of $k$, naked singularity is the only outcome. It is also to be noted that "low $\omega$ " leads to the possibility of getting more naked singularities even for a non-accelerating universe.
\end{abstract}

Pacs no: $04.20 . \mathrm{Dw}$ (Singularities and Cosmic Censorship), 98.65.D(Dark energy)

\footnotetext{
${ }^{1}$ prudra.math@gmail.com

${ }^{2}$ biswas.ritabrata@gmail.com

${ }^{3}$ ujjaldebnath@yahoo.com
} 


\section{Introduction}

Pioneered by the work of C. H. Brans, and R. H. Dicke (Brans, C. H. et. al. 1961) in 1961 one thought (rather to say a metric theory of gravitation) parallel to General Relativity (GR hereafter) was importantly coined in literature. GR considers the stress energy or matter tensor to be the source of the gravitational field. The manner in which the pressure of mass-energy acts in a region in case of Brans-Dicke theory, is completely different from the way in which it acts in case of GR. We know that in GR the geometric curvature of space-time totally controls the motion of a body. But in case of Brans-Dicke theory this dependence is somewhat alleviated. These salient features of difference is testimony of the fact that Brans-Dicke theory is quite different from the traditional theories of GR and as a result triggers a lot of research. In Brans-Dicke theory an additional scalar term $\phi$ is involved with the second ranked tensor of energy mass. This $\phi$ introduces a physical effect that changes the effective gravitational constant from place to place. Brans-Dicke's construction was however supported by the earlier works of Pascual Jordan (1959).

The field equations of Brans-Dicke theory contain a dimensionless parameter $\omega$, known as Brans-Dicke coupling constant. This is a tuneable parameter, the value of which can be adjusted to be consistent with observational evidences. Brans-Dicke theory is "less strignent" than GR in another sense: it admits more solutions. In particular, exact vacuum solutions of the Einstein field equation of GR, augmented by the trivial scalar field $\phi=1$, become exact vacuum solutions in Brans-Dicke theory. But some space times which are not vacuum solutions to the Einstein field equation become solutions for Brans-Dicke theory, with appropriate choice of scalar field, vacuum.

Like GR, Brans-Dicke theory predicts light deflection and the precession of perihelia of planets orbiting the Sun. However, the precise formulae which govern these effects, according to Brans-Dicke theory, depend upon the value of the coupling constant $\omega$. This means that it is possible to set an observational lower bound on the possible values of $\omega$ from observations of the solar system and other gravitational systems. It should be stated that the value of $\omega$ consistent with experiment has risen with time. In 1973, $\omega>5$ was consistent with known data. By 1981, $\omega>30$ was consistent with known data. Viking Space Probe says $\omega$ should exceed 500 (from timing experiments) (Reasenberg, R. D. et. al. 1979). In 2003 evidence derived from the Cassini-Huygens experiment shows that the value of $\omega$ must exceed 40,000. It is often thought that GR is obtained from the Brans-Dicke theory in the limit $\omega \rightarrow \infty$ (Barrow, J. D. et. al. 1990). But Faraoni claims that this breaks down when the trace of the stress-energy momentum vanishes, i.e. $T_{\mu}^{\mu}=0$. Some have argued that only general relativity satisfies the strong equivalence principle.

Oppenheimer and Snyder, for the first time analyzed the collapse of a dust cloud with a static Schwartzchild exterior and Friedmann like interior (Oppenhiemer, J. R. et. al. 1939). In classical GR gravitational collapse is a problem of great curiosity as we can get at least two types of singularities from it. One, covered by an event horizon, is coined as a black hole (BH hereafter) whereas the singularity alone is popular as Naked Singularity (NS hereafter). To determine the exact initial condition leading to the formation of BH or NS is a thought experiment full of challenge. Physically thinking the most important finding is a physical initial condition leading down to the formation of a NS. After all, one would always like to test the validity of cosmic censorship hypothesis (CCH) laid down by R.Penrose, (Penrose, R. 1969) which stated that the end result of a collapse is bound to be a singularity shrouded by an event horizon, i.e. a BH. Many works (Eardley, D. M. et. al. 1979; Christodoulou, D. 1984; Newman, R. P. A. C. 1986; Dwivedi, I. H. et. al. 1989; Waugh, B. et. al. 1986; Ori, A. et. al. 1990) are there in last few decades where the possibility of formation of NS has been investigated. There is no general theory of the nature on the visibility of singularities. Vaidya solution (Vaidya, P. C. 1951) is utilized on many occasions to determine the end state of collapse. Harko et al (Harko, T. et. al. 2000) have studied the gravitational collapse of strange matter and analyzed the condition for formation of a NS in the spherically symmetric Vaidya space-time. It has been shown that whether a BH or a NS will be formed, is dependent upon many issues like the initial distribution of density and velocity, the constitutive nature of the collapsing matter etc. One of the generalizations, among the many generalizations of Vaidya metric, known as the Husain solution has been used to study the formation of a BH with short hair.

Maeda (Maeda, H. 2006) started the study of spherically symmetric gravitational collapse without finding the explicit form of the solution. S. Jhingan and S. G. Ghosh (Jhingan, S. et. al. 2010) has shown that the different orders of curvature corrections can cause sensible changes in the final fate of the gravitational collapse (in the sense that massive NS is formed). This work was followed by another quasi spherical gravitational col- 
lapse in 5D Einstein Gauss Bonnet gravity (Ghosh, S. G. et. al. 2010). Recently, another study of gravitational collapse in $f(R)$ gravity was studied by S. G. Ghosh and S. D. Maharaj (Ghosh, S. G. et. al. 2012) where they have obtained a condition for the occurrence of a NS in the collapse of null dust in higher dimensional $f(R)$ gravity. T.P. Singh and P.S. Joshi in their several papers for the first time used the technique of the existence of outgoing null geodesic from the end state of collapse to identify the central singularity is a naked singularity or a singularity covered with an event horizon (Joshi, P. S. et. al. 1995; Singh, T. P. et. al. 1996; Joshi, P. S. et. al. 1992; Joshi, P. S. et. al. 1993; Lake, K. 1992; Szekeres, P. et. al. 1993; Joshi, P. S. 1993). Their method eventually became very popular and several works on the same method was done in different gravity theories and with different exotic fluids (Patil, K. D. et. al. 2005; Patil, K. D. et. al. 2006; Debnath, U. et. al. 2008; Debnath, U. et. al. 2004; Banerjee, A. et. al. 2003; Rudra, P. et. al. 2011; Debnath, U. et. al. 2012; Rudra, P. et. al. 2012). Debnath et al in (Debnath, U. et. al. 2012) and Rudra et al in (Rudra, P. et. al. 2011) have studied the end fate of collapse in higher dimensional gravity theory. They have shown that, farther one goes from Einstein gravity, there is a greater chance of having a NS. Here the effect of exotic fluid as the initial substance of the collapsing cloud has been also studied and it has been concluded that, more exotic the matter, more is the chance of having NS. Scheel in (Scheel, M. A. et. al. 1995) demonstrated that Openheimer-Snyder collapse in Brans-Dicke theory results in BHs rather than NSs (which is true for a particular range of $\omega$ ) with the positive values of $\omega$, they have speculated that the apparent horizon of a $\mathrm{BH}$ can pass outside the event horizon causing the decrease in the surface area over time. The non negative values of $\omega$ forces the $\mathrm{BH}$, to radiate its scalar mass to infinity soon after the initial collapse. Otherwise for negative $\omega$ the opposite incident occurs. In (Dong-il Hwang et. al. 2010) we get another relevant and interesting work regarding the gravitational collapse in the background of Brans-Dicke theory of gravity discussing the effects of different values of $\omega$.

Non-static spherically symmetric solutions of Einstein equations, for a null fluid source was given by Husian (Husain, V. 1996) in 1996, where the density $\rho$ and pressure $p$ of the fluid is related by $p=k \rho$. Two salient features of the solution were that it is exact and inhomogeneous in nature. In Husain's work, the $k>1 / 2$ solutions describe the evolution of a naked singularity into a black hole as the collapse proceeds. The parameters in the Vaidya metric determine which of these possibilities occur, and a black hole always forms at a finite nonzero mass. The $k<1 / 2$ solutions describe the collapse of radiation from flat space to a black hole. All of the new solutions supported the cosmic censorship conjecture. Later the Vaidya solution was generalized by Wang et al (Wang, A. et. al. 1999). The solution included most of the known solutions of the Einstein equation such as the anti-de-Sitter charged Vaidya solution. Moreover the Husain solution has been extensively used to study the formation of a black hole with short hair (Brown, J. D. et. al. 1997). The most recent development in Husain solution was witnessed when the gravitational collapse of the Husain solution in four and five dimensional space-times was studied by Patil et al (Patil , K. D. et. al. 2006).

Keeping all the previous works of gravitational collapse in GR/ different gravity theories in mind we feel it will be of a great interest if we investigate the existence of radial null geodesics from the collapsing body in the back ground of BD theory. The scalar factor present in the theory may help in collapse to form a NS more prominently than the GR does. Even we can use the BD parameter $\omega$ as a regulator and can see what happens if we deviate more and more from the Einstein gravity making $\omega$ sufficiently low. In this concern we must recall the fact that in (Rudra, P. et. al. 2011) while working with Lovelock gravity, we saw that greater the deviation from Einstein gravity greater was the tendency to have the NS. So in this paper, we are mainly studying the nature of singularities(BH or NS) formed by the gravitational collapse in Brans-Dicke theory of gravity. In section (2), we present the brief overview of generalized Husain solution in Brans-Dicke theory of gravity. We will first construct the Einstein field equations in BD theory for the Vaidya metric and then with a proper choice of the structural dependence of the potential term upon the scalar field we will determine the $m(t, r)$, the mass term. In the next two sections we investigate the behaviour/existence of the outgoing radial null geodesic from the singularity taking the Vaidya metric with the mass term $m(t, r)$ derived in the last section. Finally, the paper ends with some concluding remarks in section (5).

\section{Field Equations}

The self-interacting Brans-Dicke theory is described by the action: (choosing $8 \pi G_{0}=c=1$ ) (Chakraborty, W. et. al. 2009)

$$
S=\int d^{4} x \sqrt{-g}\left[\phi R-\frac{\omega(\phi)}{\phi} \phi^{, \alpha} \phi,_{\alpha}-V(\phi)+\mathcal{L}_{m}\right]
$$


where $V(\phi)$ is the self-interacting potential for the $\mathrm{BD}$ scalar field $\phi$ and the constant $\omega$ is the BD parameter.

Here we consider the metric in spherically symmetric space-time in the form (Vaidya, P. C. 1951)

$$
d s^{2}=-\left(1-\frac{m(t, r)}{r}\right) d t^{2}+2 d t d r+r^{2} d \Omega_{2}^{2}
$$

where $r$ is the radial co-ordinate and $t$ is the null co-ordinate, $m(t, r)$ gives the gravitational mass inside the sphere of radius $r$ and $d \Omega_{2}^{2}$ is the line element on a unit 2-sphere.

From the Lagrangian density (1) we obtain the field equations (Chakraborty, W. et. al. 2009)

$$
G_{\mu \nu}=\frac{\omega(\phi)}{\phi^{2}}\left[\phi_{, \mu} \phi_{, \nu}-\frac{1}{2} g_{\mu \nu} \phi_{, \alpha} \phi^{, \alpha}\right]+\frac{1}{\phi}\left[\phi_{, \mu ; \nu}-g_{\mu \nu} \square \phi\right]-\frac{V(\phi)}{2 \phi} g_{\mu \nu}+\frac{1}{\phi} T_{\mu \nu}
$$

and

$$
\square \phi=\frac{1}{3+2 \omega} T-\frac{1}{3+2 \omega}\left[2 V(\phi)-\phi \frac{d V(\phi)}{d \phi}\right]
$$

where $T=T_{\mu \mu} g^{\mu \mu}$.

Now we consider two types of fluids like Vaidya null radiation and a perfect fluid having the form of the energy momentum tensor

$$
T_{\mu \nu}=T_{\mu \nu}^{(n)}+T_{\mu \nu}^{(m)}
$$

with

$$
T_{\mu \nu}^{(n)}=\sigma l_{\mu} l_{\nu}
$$

and

$$
T_{\mu \nu}^{(m)}=(\rho+p)\left(l_{\mu} \eta_{\nu}+l_{\nu} \eta_{\mu}\right)+p g_{\mu \nu}
$$

Where, $\rho$ and $p$ are the energy density and pressure for the perfect fluid and $\sigma$ is the energy density corresponding to Vaidya null radiation. In the co-moving co-ordinates $\left(v, r, \theta_{1}, \theta_{2}, \ldots, \theta_{n}\right)$, the two eigen vectors of energy-momentum tensor namely $l_{\mu}$ and $\eta_{\mu}$ are linearly independent future pointing null vectors having components

$$
l_{\mu}=(1,0,0, \ldots, 0) \quad \text { and } \quad \eta_{\mu}=\left(\frac{1}{2}\left(1-\frac{m}{r^{n-1}}\right),-1,0, \ldots, 0\right)
$$

and they satisfy the relations

$$
l_{\lambda} l^{\lambda}=\eta_{\lambda} \eta^{\lambda}=0, l_{\lambda} \eta^{\lambda}=-1
$$

\section{The Solution}

The Einstein field equations $\left(G_{\mu \nu}=T_{\mu \nu}\right)$ for the metric (2) and the wave equation for the BD scalar field $\phi$ are the following

$$
\begin{gathered}
\frac{(r-m) m^{\prime}+r \dot{m}}{r^{3}}=\frac{\omega}{\phi^{2}}\left[\dot{\phi}^{2}+\frac{1}{2}\left(1-\frac{m}{r}\right)\left\{2 \dot{\phi} \phi^{\prime}+\phi^{\prime 2}\left(1-\frac{m}{r}\right)\right\}\right]+\frac{1}{\phi}\left[\ddot{\phi}-\frac{\dot{\phi}}{2 r}\left(\frac{m}{r}-m^{\prime}\right)\right. \\
\left.-\frac{\phi^{\prime}}{2 r}\left(\frac{m}{r}-\frac{m^{2}}{r^{2}}-m^{\prime}+\frac{m m^{\prime}}{r}+\dot{m}\right)+\left(1-\frac{m}{r}\right)\left\{2(\dot{\phi})^{\prime}+\phi^{\prime \prime}\left(1-\frac{m}{r}\right)-\frac{\phi^{\prime}}{r}\left(2-\frac{3 m}{r}+m^{\prime}\right)\right\}\right] \\
+\frac{V(\phi)}{2 \phi}\left(1-\frac{m}{r}\right)+\frac{1}{\phi}\left[\sigma+\rho\left(1-\frac{m}{r}\right)\right] \\
\frac{\omega \phi^{\prime 2}}{\phi^{2}}+\frac{\phi^{\prime \prime}}{\phi}=0 \\
-\frac{1}{2} r m^{\prime \prime}=\frac{\omega}{\phi^{2}}\left[-\frac{r^{2}}{2} \phi^{\prime}\left\{2 \dot{\phi}+\phi^{\prime}\left(1-\frac{m}{r}\right)\right\}\right]+\frac{1}{\phi}\left[r \dot{\phi}-m \phi^{\prime}-2 r^{2}(\dot{\phi})^{\prime}-r^{2} \phi^{\prime \prime}\left(1-\frac{m}{r}\right)+r \phi^{\prime}\left(3-3 \frac{m}{r}+m^{\prime}\right)\right] \\
-\frac{V(\phi)}{2 \phi} r^{2}+\frac{p r^{2}}{\phi}
\end{gathered}
$$




$$
-\frac{m^{\prime}}{r^{2}}=\frac{\omega}{\phi^{2}}\left[-\frac{\phi^{\prime 2}}{2}\left(1-\frac{m}{r}\right)\right]+\frac{1}{\phi}\left[-(\dot{\phi})^{\prime}+\frac{\phi^{\prime}}{r}\left(\frac{m^{\prime}}{2}-\frac{5 m}{2 r}+2\right)-\phi^{\prime \prime}\left(1-\frac{m}{r}\right)-\frac{V(\phi)}{2}-\rho\right]
$$

and

$$
2(\dot{\phi})^{\prime}+\phi^{\prime \prime}\left(1-\frac{m}{r}\right)-\phi^{\prime}\left(\frac{2}{r}-\frac{3 m}{r^{2}}+\frac{m^{\prime}}{r}\right)=\frac{\rho-3 p}{3+2 \omega}+\frac{1}{3+2 \omega}\left[2 V(\phi)-\phi \frac{d V(\phi)}{d \phi}\right]
$$

where an over-dot and dash stand for differentiation with respect to $t$ and $r$ respectively.

Here we use the power law form of potential in the Brans-Dicke theory as given below (Bisabr, Y. 2012, Chattopadhyay, S. 2013, Yang, W-Q. et al. 2011). While studying the evolution of naked singularities in Brans-Dicke cosmology, Ziaie, A. H. et al (2010) has shown that for particular matter fields if $\phi=a^{\alpha}$ then $V(\phi)$ takes the form $\beta \phi^{-\frac{3\left(1+\omega_{B D}\right)}{\alpha}}$ where $\beta=2+\frac{\alpha}{3}\left(1+\rho_{0 m}\right)\left(6+\omega_{B D}\right)$, here $\rho_{0 m}$ stands for present time barotropic mass density. Ultimately we can generalise the field dependency of the potential as

$$
V(\phi)=V_{0} \phi^{n}
$$

On solving field equation (11), the expression for $\phi$ is obtained as

$$
\phi=B(t) r^{\frac{1}{\omega+1}}
$$

where $B(t)$ is the arbitrary function of $t$. We assume the matter fluid obeys the barotropic equation of state

$$
p=k \rho, \quad(k, \text { a constant })
$$

Using equations (12), (13), (15) and (17), we have the differential equation in $m$ as

$$
\begin{aligned}
& \left(\frac{1}{2 k r}\right) m^{\prime \prime}+\left[\frac{k+2+2 k(\omega+1)}{2 r^{2} k(\omega+1)}\right] m^{\prime}-\left[\frac{6 k \omega+5 k+9 \omega+8}{2 k(\omega+1)^{2} r^{3}}\right] m \\
& +\left[\frac{5 \omega k+4 k+7 \omega+6}{2 k r^{2}(\omega+1)^{2}}-\frac{\dot{B}(1+k)}{B r k(\omega+1)}-\frac{V_{0}}{2 k}(1+k) \phi^{n-1}\right]=0
\end{aligned}
$$

Solving the above differential equation we obtain the explicit solution for $m$ as,

$$
\begin{gathered}
m(t, r)=f_{1}(t) r^{\omega_{1}}+f_{2}(t) r^{\omega_{2}}+\frac{5 \omega k+4 k+7 \omega+6}{\left(1-\omega_{1}\right)\left(1-\omega_{2}\right)} r-\frac{2 \dot{B}(1+k)(1+\omega)}{B\left(2-\omega_{1}\right)\left(2-\omega_{2}\right)} r^{2} \\
-\frac{V_{0} B^{n-1}(\omega+1)^{4}(k+1)}{\left\{(\omega+n)-\omega_{1}(\omega+1)\right\}\left\{(\omega+n)-\omega_{2}(\omega+1)\right\}} r^{\frac{\omega+n}{\omega+1}}
\end{gathered}
$$

where

$$
\omega_{1}, \omega_{2}=\frac{(\omega-3 k-2 k \omega-1) \pm \sqrt{\{(k+2)+(2 k-1)(\omega+1)\}^{2}-4(6 k \omega+5 k+9 \omega+8)}}{2(\omega+1)}
$$

Here $f_{1}(t)$ and $f_{2}(t)$ are arbitrary functions of $t$.

Therefore the metric (2) can be written as

$$
\begin{aligned}
d s^{2} & =\left[1-f_{1}(t) r^{\omega_{1}-1}-f_{2}(t) r^{\omega_{2}-1}-\frac{5 \omega k+4 k+7 \omega+6}{\left(1-\omega_{1}\right)\left(1-\omega_{2}\right)}+\frac{2 \dot{B}(1+k)(1+\omega)}{B\left(2-\omega_{1}\right)\left(2-\omega_{2}\right)} r\right. \\
& \left.+\frac{V_{0} B^{n-1}(\omega+1)^{4}(k+1)}{\left\{(\omega+n)-\omega_{1}(\omega+1)\right\}\left\{(\omega+n)-\omega_{2}(\omega+1)\right\}} r^{\frac{n-1}{\omega+1}}\right] d t^{2}+2 d t d r+r^{2} d \Omega_{2}^{2}
\end{aligned}
$$

which is called the the Husain metric or Generalized Vaidya metric in Brans-Dicke gravity. 


\section{Collapse Study}

We shall discuss the existence of NS in generalized Vaidya space-time by studying radial null geodesics. In fact, we shall examine whether it is possible to have outgoing radial null geodesics which were terminated in the past at the central singularity $r=0$. The nature of the singularity (NS or $\mathrm{BH}$ ) can be characterized by the existence of radial null geodesics emerging from the singularity. The singularity is at least locally naked if there exist such geodesics and if no such geodesics exist it is a $\mathrm{BH}$.

A singularity caused by a catastrophic gravitational collapse may be a NS or a BH. Famous Hawking-Penrose singularity theorems provide no information on that issue. The cosmic censorship hypothesis essentially states that , in GR, the end state of gravitational collapse is always a BH : the gravitational singularity must necessarily be covered by an event horizon. However, it comes with no known proof. Inhomogeneous dust cloud may result in a naked singularity through a collapse (Eardley, D. M. et. al. 1979). Fluids with different EoS other than dust also give rise to interesting results (Joshi, P. S. et. al. 1992). Keeping the above literature in view the censorship hypothesis needs to get generalised (Joshi, P. S. et. al. 1995).

Let $R(t, r)$ is the physical radius at time $t$ of the shell labelled by $r$. Scaling freedom says at the starting epoch $t=0$ we should have $R(0, r)=r$. We should keep it in mind that in the inhomogeneous case (more generalized one), different shells become singular at different times. Now if there are future directed radial null geodesics coming out of the singularity, with a well defined tangent at the singularity $\frac{d R}{d r}$ must tend to a finite limit in the limit of approach to the singularity in the past along these trajectories.

The points $\left(t_{0}, r\right)=0$ occurs, where the singularity $R\left(t_{0}, 0\right)=0$ occurs corresponds to the physical situation where matter shells are crushed to zero radius. This kind of singularity $(r=0)$ is known to be a central singularity.

The singularity is an NS if there are future directed non-space like curves in the space time with their past end points at the singularity. Now if the outgoing null geodesics are to terminate in the past at the central singularity at $r=0$ at $t=t_{0}$ where $R\left(t_{0}, 0\right)=0$, then along these geodesics we should have (Singh, T. P. et. al. 1996) $R \rightarrow 0$ as $r \rightarrow 0$.

The equation for outgoing radial null geodesics can be obtained from equation (2) by putting $d s^{2}=0$ and $d \Omega_{2}^{2}=0$ as

$$
\frac{d t}{d r}=\frac{2}{\left(1-\frac{m(t, r)}{r}\right)} .
$$

It can be seen easily that $r=0, t=0$ corresponds to a singularity of the above differential equation. Suppose $X=\frac{t}{r}$ then we shall study the limiting behavior of the function $X$ as we approach the singularity at $r=0, t=0$ along the radial null geodesic. If we denote the limiting value by $X_{0}$ then

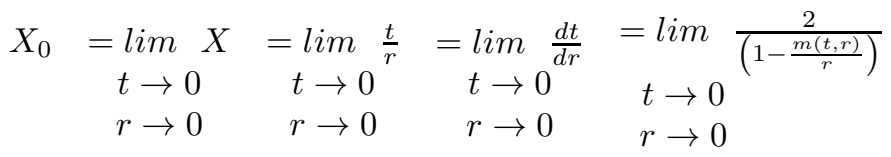

Using equations (19) and (23), we have

$$
\begin{gathered}
\frac{2}{X_{0}}=\lim _{\substack{r \rightarrow 0 \\
r \rightarrow 0}}\left[1-f_{1}(t) r^{\omega_{1}-1}-f_{2}(t) r^{\omega_{2}-1}-\frac{5 \omega k+4 k+7 \omega+6}{\left(1-\omega_{1}\right)\left(1-\omega_{2}\right)}+\frac{2 \dot{B}(1+k)(1+\omega)}{B\left(2-\omega_{1}\right)\left(2-\omega_{2}\right)} r\right. \\
\left.+\frac{V_{0} B^{n-1}(\omega+1)^{4}(k+1)}{\left\{(\omega+n)-\omega_{1}(\omega+1)\right\}\left\{(\omega+n)-\omega_{2}(\omega+1)\right\}} r^{\frac{n-1}{\omega+1}}\right]
\end{gathered}
$$

Now choosing $f_{1}(t)=\lambda t^{-\left(\omega_{1}-1\right)}, \quad f_{2}(t)=\gamma t^{-\left(\omega_{2}-1\right)}$ and $B(t)=B_{0} t^{-\frac{1}{1+\omega}}$, we obtain the algebraic equation of $X_{0}$ as

$$
\begin{gathered}
\frac{V_{0}(\omega+1)^{4}(k+1) B_{0}^{n-1}}{\left[(\omega+n)-\omega_{1}(\omega+1)\right]\left[(\omega+n)-\omega_{2}(\omega+1)\right]} X_{0}^{\frac{\omega-n+2}{\omega+1}}-\lambda X_{0}^{2-\omega_{1}}-\gamma X_{0}^{2-\omega_{2}} \\
+\left[1-\frac{5 \omega k+4 k+7 \omega+6}{\left(1-\omega_{1}\right)\left(1-\omega_{2}\right)}\right] X_{0}-2\left[1+\frac{(1+k)}{\left(2-\omega_{1}\right)\left(2-\omega_{2}\right)}\right]=0
\end{gathered}
$$

Now if we get only non-positive solution of the equation we can assure the formation of a BH. Getting a positive root indicates a chance to get a NS. Since the obtained equation is a highly complicated one, it is extremely 
difficult to find out an analytic solution of $X_{0}$ in terms of the variables involved. So our idea is to find out different numerical solutions of $X_{0}$, by assigning particular numerical values to the associated variables.

The different solutions of $X_{0}$ for different sets of parametric values $\left(\lambda, \gamma, V_{0}, B_{0}, n, \omega, k\right)$ are given here in a tabular form (Table 1a-e).

\begin{tabular}{|c|c|c|c|c|c|}
\hline & & & \multicolumn{3}{|c|}{ For $k=1$ (stiff perfect fluid) } \\
\hline $\bar{\omega}$ & & & $\overline{\overline{V_{0}}}$ & $\overline{\overline{B_{0}}}$ & roots $\left(X_{0}\right)$ \\
\hline 2 & 1 & 1 & 5 & 6 & - \\
\hline " & & , & , & ", & - \\
\hline 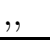 & 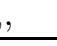 & , & & ", & - \\
\hline 1 & 0.1 & 0.1 & 1 & 1 & - \\
\hline, & , & , & , & , & - \\
\hline , & 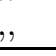 & 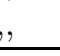 & 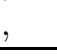 & , & - \\
\hline-2 & 0.1 & 0.1 & 1 & 1 & - \\
\hline , & , & , & , & , & 0.9449257 \\
\hline , & , & 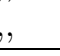 & 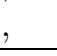 & , & 0.8592578 \\
\hline-3 & 0.1 & 0.1 & 1 & 1 & - \\
\hline " & , & ", & & , & 0.4381 \\
\hline , & , & , & , & , & 0.39825 \\
\hline
\end{tabular}

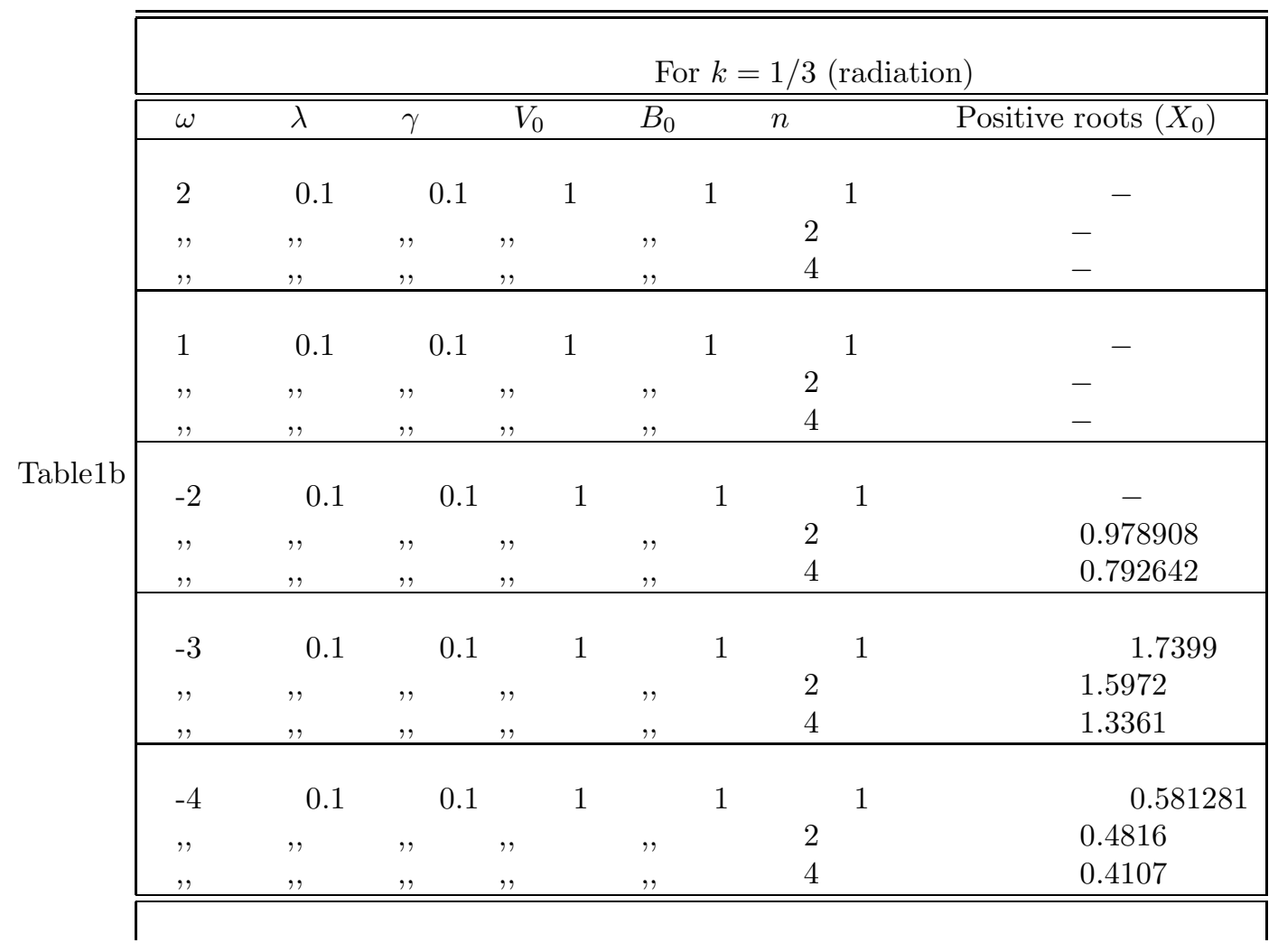




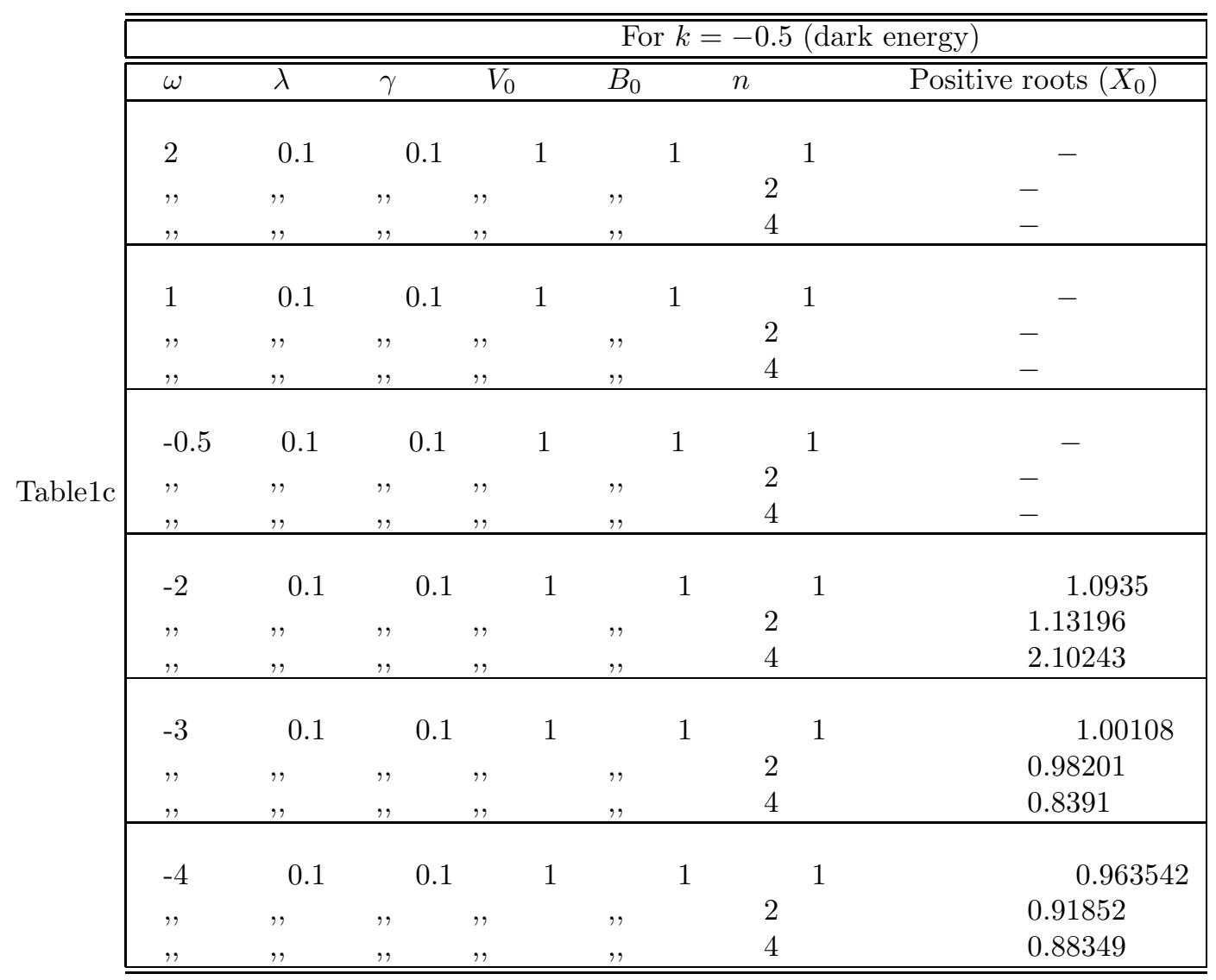

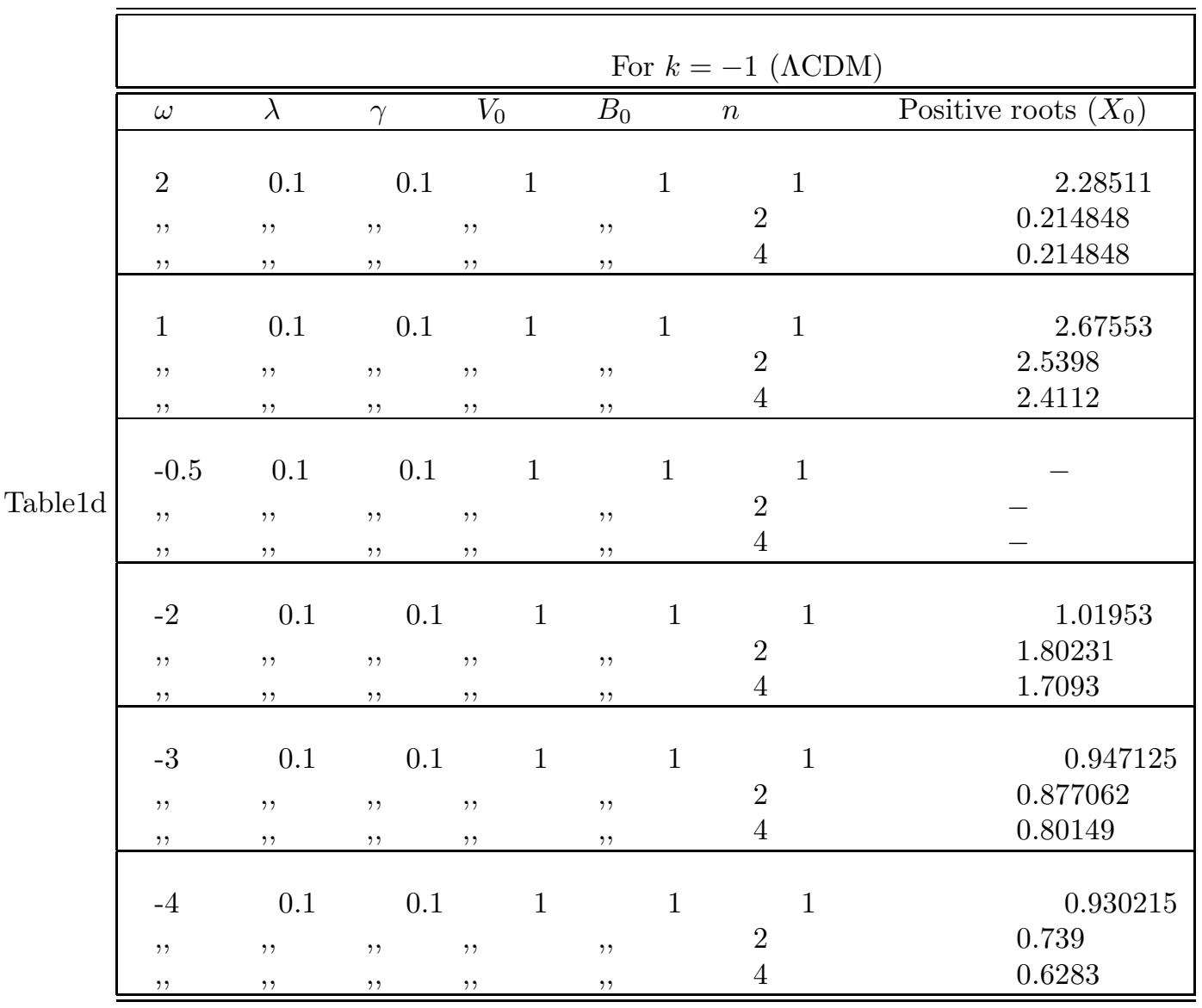




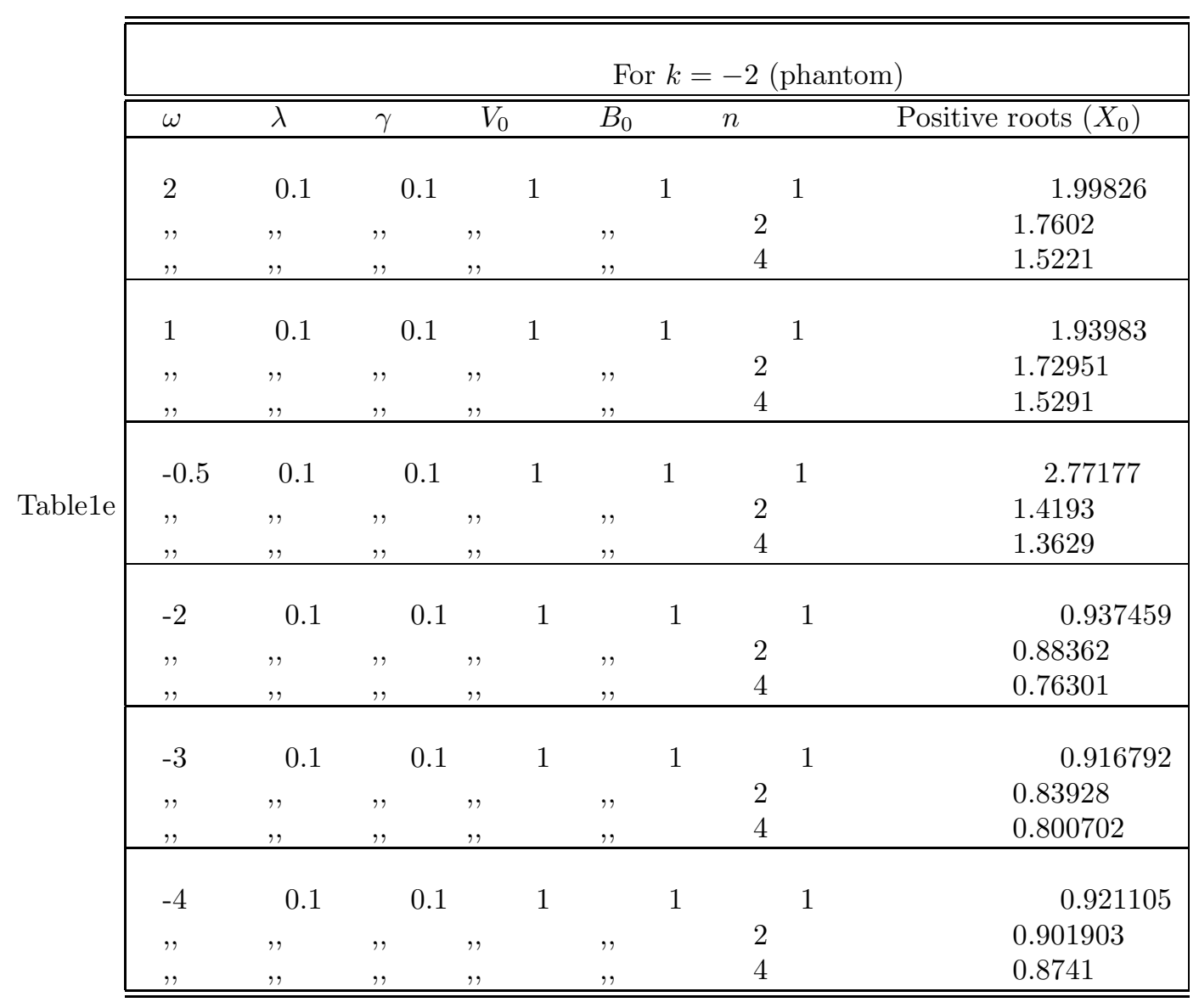

Table 1a-e: Values of $X_{0}$ for different values of parameters $\lambda, \gamma, V_{0}, B_{0}, n, \omega, k$.

Here, the two very important parameters are $\omega$, the Brans-Dicke parameter and the EoS parameter, $k$. With a slight variation of all other parameters we have considered two extremal variations for $\omega$ and $k$. For EoS parameter $k$ being positive $(=1)$ one we get no positive solution irrespective of the value of the $\omega$ in the range $-3<\omega<2$ if $n$ is considered to be unity. But for higher values of $n$ there is a possibility of having positive solutions for highly negative $\omega$ cases, i.e., for greater deviations from Einstein gravity. But highly negative values of $\omega$ are not physical either, since they produce ghost. In radiation era $\left(k=\frac{1}{3}\right)$, for highly negative values of $\omega$ positive roots are found. However, $\omega>-2$ contributes no positive solutions in the radiation era. In expanding universe the same trend continues except the fact that here the lower limit of the range of $\omega$ that gives non positive solution rises compared to the radiation era. At phantom crossing and phantom era we always have positive solutions (for the range $-4<\omega$ ). Physically interpreting we can say when positive pressure fluids are there in the universe the outcome of a collapse is more likely to be a NS. Now if we lower the value of the Brans Dicke parameter, i.e., we go far from the Einstein gravity the chances of having a NS increases even for a non-expanding universe. In fact negative values of $\omega$ almost confirms the possibility of having a NS. Leaving the positive pressure zone behind as we look into the quintessence/ phantom era i.e. we consider the expanding universe (rather to say the scenario of cosmic acceleration) it immediately gives NS as the only final fate of the collapse. Here, even though we increase $\omega$ to a notable higher value (i.e., we move towards the Einstein gravity) yet the collapse results in NS only. As only two parameters are controlling the end fate, we will plot their variations in the fig $1 a-i$. In figures 1a to $1 \mathrm{i}$ we have plotted the $k-X_{0}$ contours for increasing values of $\omega .0<k<1$ zone is less probable zone to have a contour for negative values of $\omega$. But with positive and higher values of $\omega$ contours are there over the whole range of $k$. Now whatever be the value of $\omega$ we get contours for negative $k$-s. 
Fig.1a

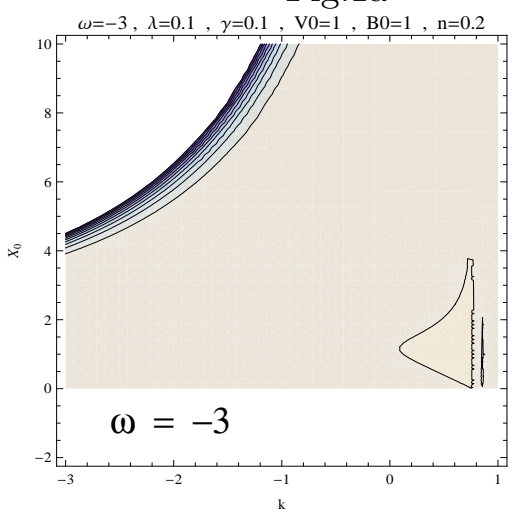

Fig.1d

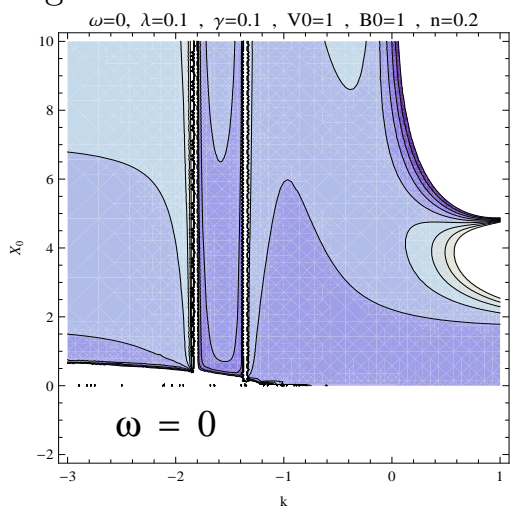

Fig.1g

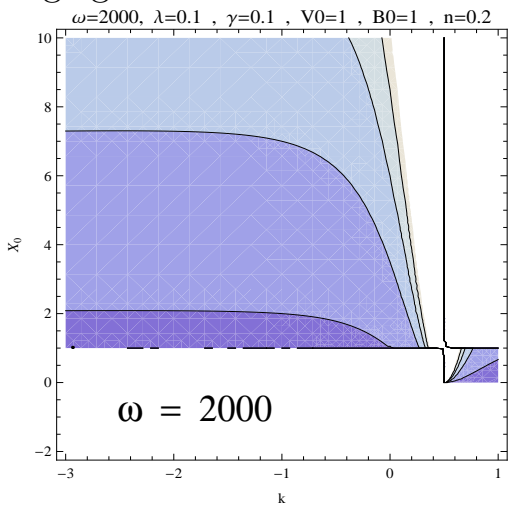

Fig.1b

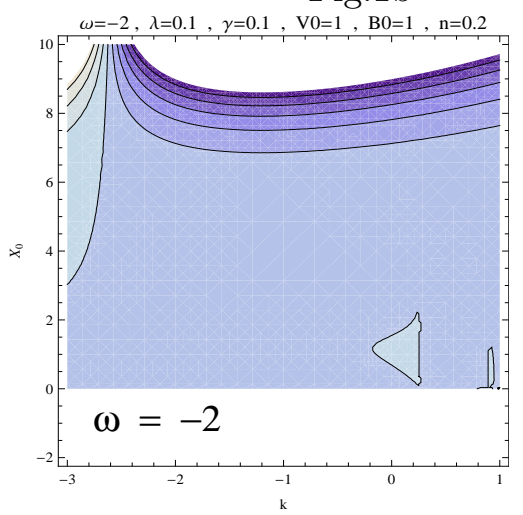

Fig.1e

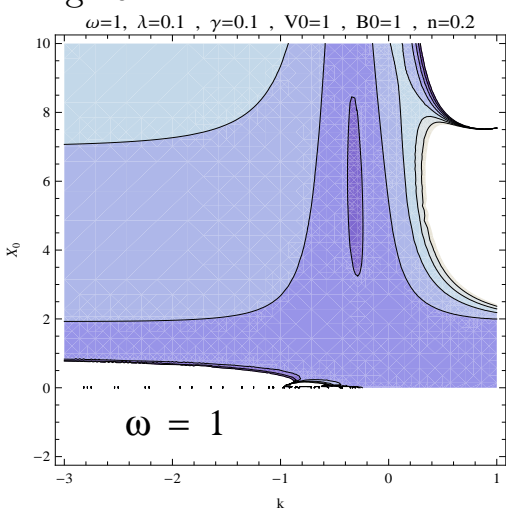

Fig.1h

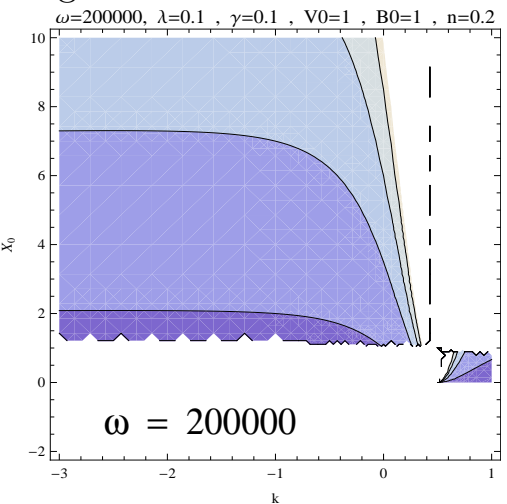

Fig.1c

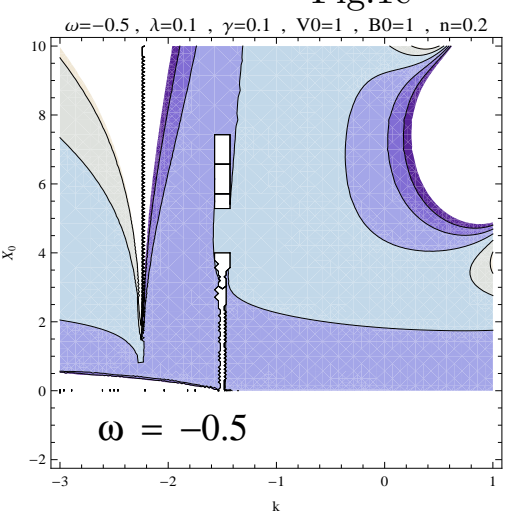

Fig.1f

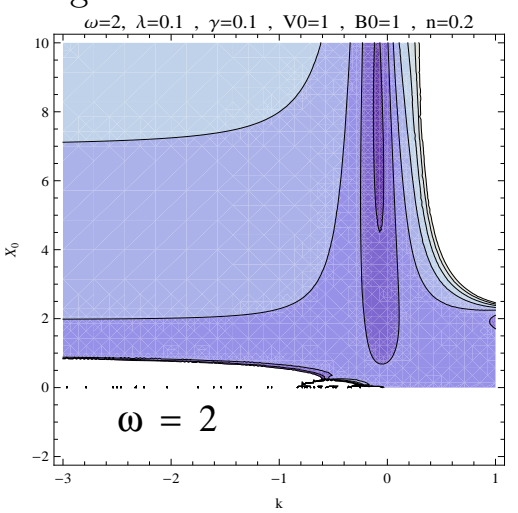

Fig.1i

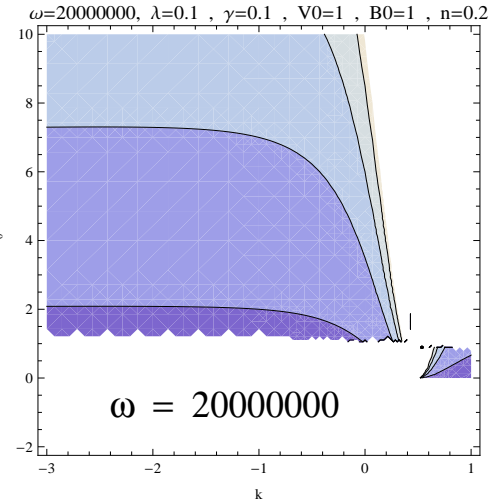

Figs. 1a-i show the $X_{0}$ contours in the $k$ - $X_{0}$ plane for different values of $\omega$. 


\section{Conclusion}

In this work, we have assumed the spherically symmetric space time model with Vaidya null radiation and perfect fluid. We have determined the solution of Einstein equation in Brans-Dicke gravity with self interacting potential and after choosing power law form of the potential we have determined the Husain metric (generalized Vaidya metric). Next step was to inspect the existence of the radial null geodesic from the final fate of the collapsing object. Existence of such geodesic points corresponds to the chances of having a NS. If not, then the possibility of a $\mathrm{BH}$ is confirmed. In this study the general trend is to have a NS if we consider late time universe and smaller values of Brans-Dicke coupling constant. Higher positive values of $\omega$ correspond to lesser deviation from Einstein gravity, whereas the lower negative values points towards extreme deviations and greater modification of the gravity theory. So when we decrease $\omega$ the probability of having a NS, as an end state of collapse becomes greater, even if a non-accelerating scenario is considered. It should be realized that sufficiently small $|\omega|$ turns the Brans-Dicke field sufficiently dynamic giving such an outcome. The trend of collapse for late time non-positive values of $k$ matches with the work of Scheel (Scheel, M. A. et al. 1995). The potential working around a compact object increases the speed of the flow around it and gradually makes it supersonic via sonic point crossing. Particularly the pseudo-Newtonian potential working around the object makes the velocity of the flow equal to the speed of light near the event horizon in case of a black hole since it does not have any hard surface like neutron stars. But in case of a NS the flow will not get absorbed at any horizon and near the singularity the flow will be exotic showing abrupt/non-uniform spectra. Such sources may be found in the cases of AGNs. Kovacs, Z., Harko, T. (2010) have proposed such a phenomenon. A similar topic was discussed by Virbhadra, K. S., Keeton, C. R. (2008) where they concluded that the lensing characteristics of strongly naked singularities are qualitatively very different from those due to Schwarzschild black holes. In this context, it is worth mentioning that if it is possible to test or compare the model described in the present assignment with the methods described in the above mentioned references, then it will be of interest to discuss their origin and may be our current results will get a stronger base and some astrophysical support. For the time being we keep it an open question worthy of addressing in near future.

\section{Acknowledgement :}

Authors thank IUCAA, Pune for local hospitality and research facilities, where a part of this work was done. RB thanks CSIR for awarding Research Associate fellowship and UD thanks CSIR project "Dark Energy Models and Accelerating Universe" (No. 03(1206)/12/EMR - II). The authors also thank the anonymous referee for his or her constructive comments that helped them to improve the quality of the manuscript.

\section{Appendix}

Equation (18) is a second order Non-homogeneous Ordinary differential equation with variable coefficient (r). If we multiply eqn. (18) by $r^{3}$, we see that it reduces to Cauchy-Euler differential equation. So we make the substitution,

$$
r=e^{z}
$$

This transforms the equation into a second order differential equation with constant coefficients, which of course can be solved by the known methods. We take the corresponding homogeneous differential equation and find the Complementary function (C.F.) as given below,

$$
\text { C.F. }\left(m_{c}\right)=f_{1}(t) r^{\omega_{1}}+f_{2}(t) r^{\omega_{2}}
$$

where

$$
\omega_{1}, \omega_{2}=\frac{(\omega-3 k-2 k \omega-1) \pm \sqrt{\{(k+2)+(2 k-1)(\omega+1)\}^{2}-4(6 k \omega+5 k+9 \omega+8)}}{2(\omega+1)}
$$


Here $f_{1}(t)$ and $f_{2}(t)$ are arbitrary functions of $t$ as stated earlier. Then we go on to calculate the Particular Integral (P.I.) for the non-homogeneous part of the differential equation as given below,

P.I. $\left(m_{p}\right)=\frac{5 \omega k+4 k+7 \omega+6}{\left(1-\omega_{1}\right)\left(1-\omega_{2}\right)} r-\frac{2 \dot{B}(1+k)(1+\omega)}{B\left(2-\omega_{1}\right)\left(2-\omega_{2}\right)} r^{2}-\frac{V_{0} B^{n-1}(\omega+1)^{4}(k+1)}{\left\{(\omega+n)-\omega_{1}(\omega+1)\right\}\left\{(\omega+n)-\omega_{2}(\omega+1)\right\}} r^{\frac{\omega+n}{\omega+1}}$

Finally we add the C.F. $\left(m_{c}\right)$ and P.I. $\left(m_{p}\right)$ to get the final expression for $m$ in eqn. 19, i.e. $m=m_{c}+m_{p}$.

\section{References}

Banerjee, A.,Debnath, U., Chakraborty, S. :- Int.J.Mod.Phys. D12 1255 (2003).

Barrow, J. D., Maeda, K. :- Nucl Phys B 341294 (1990).

Bisabr, Y. :- Phys. Rev. D 86127503 (2012).

Brans, C. H., Dicke, R. H. :- Physical Review 124925 (1961).

Brown, J. D., Husain, V. :- Int. J. Mod. Phys. D 6563 (1997).

Chakraborty, W. and Debnath, U. :- Int. J. Theor. Phys. 48232 (2009).

Chattopadhyay, S. :- ISRN High Energy Physics Article Id: 414615 (2013).

Christodoulou, D. : Commun. Math. Phys. 93171 (1984)

Debnath, U., Chakraborty, S., Barrow, J. D. : Gen.Rel.Grav. 36231 (2004)

Debnath, U., Chakraborty, N. C., Chakraborty, S. : Gen.Rel.Grav. 40749 (2008)

Debnath, U., Rudra, P., Biswas, R. :- Astrophys Space Sci 339135 (2012)

Dong-il Hwang, Dong-han Yeom :- Class.Quant.Grav. 27205002 (2010).

Dwivedi, I. H. and Joshi, P. S. : Class. Quantum. Grav. 61599 (1989)

Eardley, D. M. and Smar, L. : Phys. Rev. D 192239 (1979)

Ghosh, S.G. and Jhingan, S. :- Phys. Rev. D 82, 024017 (2010).

Ghosh, S. G., Maharaj, S. D. :- Phys. Rev. D 85, 124064 (2012).

Harko, T., Cheng, K. S. : Phys. Lett. A 266249 (2000).

Husain, V. :- Phys. Rev. D 53 R1759 (1996).

Jhingan, S., S. G. Ghosh :- Phys. Rev. D 81024010 (2010).

Joshi, P. S., Dwivedi, I. H. : Commun. Math. Phys. 146333 (1992)

Joshi, P. S., Dwivedi,I. H. : Lett. Math. Phys. 27235 (1993)

Joshi, P. S., Singh, T. P. : Phys.Rev. D 516778 (1995).

Joshi, P. S.: Global aspects in gravitation and cosmology,Clarendon Press, OUP, Oxford (1993), Chapters

6 and 7.

Kovacs, Z., Harko, T. :- Phys.Rev.D 82124047 (2010).

Lake, K. : Phys. Rev. Lett. 68, 3129 (1992)

Maeda, H. :- Phys. Rev. D 73104004 (2006).

Newman, R. P. A. C. : Class. Quantum. Grav. 3527 (1986)

Oppenhiemer, J. R., Snyder, H. : Phys. Rev. 56455 (1939).

Ori, A., Piran, T. : Phys. Rev. D 42, 1068 (1990).

Patil, K. D., Thool, U. S. : Int. J. Mod. Phys. D 14873 (2005)

Patil , K. D., Zade, S. S. I : nt. J. Mod. Phys. D 151359 (2006).

Penrose, R. : Riv Nuovo. Cimento. 1252 (1969).

Reasenberg, R. D. et. al. : ApJ 234 L219 (1979).

Rudra, P., Biswas, R., Debnath, U. :- Astrophys Space Sci 335, 505 (2011)

Rudra, P., Biswas, R., Debnath, U. :- Astrophys Space Sci 342, 557 (2012).

Scheel, M. A. et al. :- Phys. Rev. D 51 4236(1995).

Singh, T. P., Joshi, P. S. : Class.Quant.Grav. 13559 (1996).

Szekeres, P., Iyer, V.: Phys. Rev. D 47, 4362 (1993).

Vaidya, P. C. : Proc. Indian Acad. Sci., Sect. A 33264 (1951).

Virbhadra, K. S., Keeton, C. R. :- Phys. Rev. D 77124014 (2008).

Wang, A., Wu, Y. :- Gen. Rel. Grav. 31107 (1999).

Waugh, B., Lake, K. : Phys. Rev. D 34 2978(1986).

Yang, W-Q. et al. :- Mod. Phys. Lett. A 26191 (2011). 
Ziaie, A.H., Atazadeh, K., Tavakoli, Y. :- Class.Quant.Grav. 27075016 (2010). 